\title{
Probing the Hofmeister series beyond water: Specific-ion effects in non-aqueous solvents
}

Virginia Mazzini, Guangming Liu, and Vincent S. J. Craig

Citation: The Journal of Chemical Physics 148, 222805 (2018); doi: 10.1063/1.5017278

View online: https://doi.org/10.1063/1.5017278

View Table of Contents: http://aip.scitation.org/toc/jcp/148/22

Published by the American Institute of Physics

\section{Articles you may be interested in}

The influence of polarizability and charge transfer on specific ion effects in the dynamics of aqueous salt solutions

The Journal of Chemical Physics 148, 222803 (2018); 10.1063/1.5012682

Molecular dynamics study of structure and vibrational spectra at zwitterionoic lipid/aqueous $\mathrm{KCl}, \mathrm{NaCl}$, and $\mathrm{CaCl}_{2}$ solution interfaces

The Journal of Chemical Physics 148, 222801 (2018); 10.1063/1.5006543

A relationship between the effect of uni-univalent electrolytes on the structure of water and on its volatility The Journal of Chemical Physics 148, 222807 (2018); 10.1063/1.5009311

Calcium ions in aqueous solutions: Accurate force field description aided by ab initio molecular dynamics and neutron scattering

The Journal of Chemical Physics 148, 222813 (2018); 10.1063/1.5006779

Absolute proton hydration free energy, surface potential of water, and redox potential of the hydrogen electrode from first principles: QM/MM MD free-energy simulations of sodium and potassium hydration The Journal of Chemical Physics 148, 222814 (2018); 10.1063/1.5000799

2D nanoporous membrane for cation removal from water: Effects of ionic valence, membrane hydrophobicity, and pore size

The Journal of Chemical Physics 148, 222804 (2018); 10.1063/1.5013926

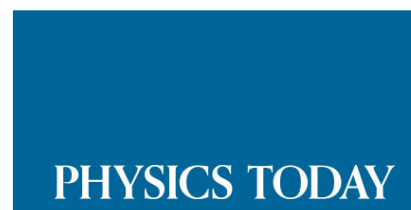

WHITEPAPERS
ADVANCED LIGHT CURE ADHESIVES

Take a closer look at what these environmentally friendly adhesive systems can do

\section{READ NOW}

PRESENTED BY

Q MASTERBOND: 


\title{
Probing the Hofmeister series beyond water: Specific-ion effects in non-aqueous solvents
}

\author{
Virginia Mazzini, ${ }^{1}$ Guangming Liu, ${ }^{2}$ and Vincent S. J. Craig ${ }^{1, a)}$ \\ ${ }^{1}$ Department of Applied Mathematics, Research School of Physics and Engineering, The Australian \\ National University, Canberra, ACT 2601, Australia \\ ${ }^{2}$ Department of Chemical Physics, Hefei National Laboratory for Physical Sciences at the Microscale, \\ University of Science and Technology of China, Hefei 230026, People's Republic of China
}

(Received 27 November 2017; accepted 19 January 2018; published online 14 February 2018)

\begin{abstract}
We present an experimental investigation of specific-ion effects in non-aqueous solvents, with the aim of elucidating the role of the solvent in perturbing the fundamental ion-specific trend. The focus is on the anions: $\mathrm{CH}_{3} \mathrm{COO}^{-}>\mathrm{F}^{-}>\mathrm{Cl}^{-}>\mathrm{Br}^{-}>\mathrm{I}^{-}>\mathrm{ClO}_{4}^{-}>\mathrm{SCN}^{-}$in the solvents water, methanol, formamide, dimethyl sulfoxide (DMSO), and propylene carbonate (PC). Two types of experiments are presented. The first experiment employs the technique of size exclusion chromatography to evaluate the elution times of electrolytes in the different solvents. We observe that the fundamental (Hofmeister) series is observed in water and methanol, whilst the series is reversed in DMSO and PC. No clear series is observed for formamide. The second experiment uses the quartz crystal microbalance technique to follow the ion-induced swelling and collapse of a polyelectrolyte brush. Here the fundamental series is observed in the protic solvents water, methanol, and formamide, and the series is once again reversed in DMSO and PC. These behaviours are not attributed to the protic/aprotic nature of the solvents, but rather to the polarisability of the solvents and are due to the competition between the interaction of ions with the solvent and the surface. A rule of thumb is proposed for ion specificity in non-aqueous solvents. In weakly polarisable solvents, the trends in specific-ion effects will follow those in water, whereas in strongly polarisable solvents the reverse trend will be observed. Solvents of intermediate polarisability will give weak specific-ion effects. Published by AIP Publishing. https://doi.org/10.1063/1.5017278
\end{abstract}

\section{INTRODUCTION}

Electrolyte solutions are ubiquitous in nature and technology. Beyond setting the ionic strength of a solution, the type of ions present is decisive in defining the properties and behaviour of such solutions. These phenomena that extend beyond the effect of ion concentration and valency are collectively called specific-ion effects and are also known as Hofmeister effects or the lyotropic series. Despite the obvious importance of specific-ion effects, a definitive theory that is capable of predicting the properties of an ionic solution from the concentration and type of the constituent ions has not yet been completed, despite recent major advances. The challenge is significant as it is extremely difficult to consistently account for all the contributions to the solvation of an ion and the interaction of an ion with other species in solution and interfaces. Despite the complexity of the matter, substantial progress is being made. ${ }^{1-3}$ This is reflected in a recent report on the current state of research in the field. ${ }^{4}$ Naturally, the vast majority of experimental reports of specific-ion effects involve aqueous solutions. However, specific-ion effects have been demonstrated in a wide range of solvents, suggesting that they occur in every solvent. ${ }^{5-12}$

The study of specific-ion effects in non-aqueous liquids has obvious relevance to applications in ion exchange,

a)vince.craig@anu.edu.au electrolysis, and energy storage systems. A particular area in which ion specificity may play a role in the future is the use of modified proteins for use in non-aqueous solvents. ${ }^{13}$ Our interest here focuses on unravelling the role of the solvent in ion specificity, with the aim of elucidating the principal properties of the solvent that govern the manifestation of ion specificity.

Furthermore, experimental results obtained using nonaqueous solvents provide a stringent testing ground for theories and explanations of specific-ion effects. For example, the importance of the protic property of water in ion specificity can be better understood by comparison with ion specificity in other solvents both protic and aprotic.

Our recent work focuses on specific-ion effects in nonaqueous solvents, and our approach has been to systematically investigate one ion-specific property in different solvents, in order to understand if and how the solvent affects the manifestation of ion specificity. An analysis of the literature on electrolyte solutions ${ }^{11,12}$ has demonstrated that whilst the manifestations of specific-ion effects across different solvents for a wide range of experiments form a very complex picture, there is an underlying simplicity. When we consider the properties of electrolyte solutions at infinite dilution, we have found that there is a fundamental ordering of specific-ion effects across all the solvents which follows a Hofmeister series for anions and a reverse-lyotropic series for cations. ${ }^{12}$ This fundamental ordering is valid in the idealised conditions of infinite dilution and for bulk properties of electrolyte solutions. 
This ordering is independent of the proticity of the solvent and demonstrates that ion-specificity arises from the ions themselves and does not require ion-ion interactions. The ionspecific trends observed under common circumstances such as finite concentrations and in the presence of interacting surfaces can be viewed as perturbations to the fundamental series. $^{12}$

Our aim here is to experimentally investigate the specificion effects manifest in non-aqueous solvents to understand how the solvent influences the relative strength of specificion effects at finite concentrations and how this varies from the ordering of ion specificity at infinite dilution. We have restricted our investigations to a set of monovalent anions of the Hofmeister series, and we have chosen sodium as the common cation. The anions we investigated are listed here according to the fundamental (i.e., Hofmeister) series,

$$
\mathrm{CH}_{3} \mathrm{COO}^{-}>\mathrm{F}^{-}>\mathrm{Cl}^{-}>\mathrm{Br}^{-}>\mathrm{I}^{-}>\mathrm{ClO}_{4}^{-}>\mathrm{SCN}^{-} \text {. }
$$

Our experiments investigate how the ordering of this anion series changes when the solvent is changed in different experiments.

We have chosen to study five solvents, three of which are protic_capable of hydrogen-bonding-water, methanol, $(\mathrm{MeOH})$, and formamide (FA) and the remaining two are aprotic, dimethyl sulfoxide (DMSO) and propylene carbonate (PC). These solvents span a wide range of properties (see Table I). Whereas we have shown ${ }^{12}$ that proticity does not matter at infinite dilution, we wish to examine if proticity matters at the salt concentrations encountered in experiments and applications, particularly as hydrogen bonding has been indicated as one of the fundamental enablers of specific-ion effects. ${ }^{14}$

We have attempted a number of different experiments, but only two of them have been successful and workable for a range of solvents and ions. These are Size Exclusion Chromatography (SEC) of electrolyte solutions on Sephadex $®$ G-10 and the investigation of the conformation of a polymer brush in the presence of the different electrolytes by Quartz Crystal Microbalance with Dissipation (QCM-D). The other experiments that we attempted without success include monitoring the flocculation of Boehmite colloids by turbidimetry, the influence of electrolytes on the NMR $T_{2}$ relaxation times of water, and the swelling of a hydrogel in a range of electrolyte solutions. The reasons for such a low rate of success are that for these experiments to yield useful results, the experiment must operate in solvents with a wide range of properties, a range of salts of sufficient solubility across the solvents have to be identified, and the effect one is trying to measure must be detectable below the solubility limit of a wide range of electrolytes. Additionally the materials used in the experiment and in the apparatus must be compatible with the different solvents. Most experimental work is performed on aqueous solutions, and therefore most experimental apparatuses are not designed or have not been tested for use with a wide variety of solvents. Furthermore, as this work has progressed, we have become acutely aware that our intuition is mainly developed from experience with aqueous solutions. As such we tend to implicitly assume that approaches that are effective for investigating aqueous electrolytes will also prove effective with non-aqueous electrolytes, which experience tells us is often not true. Perhaps of more concern is that this false intuition might lead us to fail to consider approaches that might be fruitful in non-aqueous solvents that are not commonly used in aqueous systems

\section{A. Precedents to these experiments}

Preceding experimental work on specific-ion effects in aqueous systems influenced the choice of experiments. An important contribution in the study of specific-ion effects is the characterisation of electrolytes of biological interest by size exclusion chromatography, performed by Washabaugh and Collins. ${ }^{21}$ The ion specificity observed in these experiments was the opposite of what would be expected if the ions in solution behaved ideally. Ideal ions are expected to interact with their hydration shells with the same strength (independently of the ion type), and they are expected not to interact with the columns (uncharged) stationary phase. In ideal conditions, small electrolytes are expected to elute in order of decreasing size and to leave the column after the void volume, but before one column volume of eluent has been passed (see Table II for the size of the molecules). As the ions investigated are of similar size to the eluent, it is expected that their retention factor would be close to 1 (the eluent retention factor).

Rather, Washabaugh and Collins found that the electrolytes do not behave ideally and are eluted in order of increasing size, in agreement with the Hofmeister series. In addition, some of the electrolytes elute later than one column volume of eluent. The explanation proposed was that small anions such as fluoride are strongly hydrated and travel in solution with their

TABLE I. Physical properties of the solvents used in this study. ${ }^{a}$

\begin{tabular}{lllllll}
\hline \hline Solvent & $\rho\left(\mathrm{g} \mathrm{ml}^{-1}\right)$ & $\varepsilon_{\mathrm{R}}$ & $\mu(\mathrm{D})$ & $\eta(\mathrm{mPas})$ & $\alpha\left(\AA^{3}\right)$ & H-bonding \\
\hline Water & $0.9970^{25}$ & 80.100 & 1.856 & 0.890 & 1.49 & 3D H-bond \\
MeOH & 0.7914 & 33.0 & 1.7 & 0.544 & 3.26 & Linear H-bond \\
FA & 1.1334 & 111.0 & 3.73 & 3.34 & 4.22 & 3D H-bond \\
DMSO & $1.1010^{25}$ & 47.24 & 3.96 & 1.987 & 8.03 & Aprotic \\
PC & 1.2047 & 66.14 & $5.36^{\mathrm{b}}$ & $2.5120^{\mathrm{c}}$ & 8.55 & Aprotic \\
\hline \hline
\end{tabular}

${ }^{\mathrm{a}} \rho$ : density at $20{ }^{\circ} \mathrm{C}$ unless otherwise indicated by the superscript; $\varepsilon_{\mathrm{R}}$ : dielectric constant; $\mu$ : dipole moment; $\eta$ : viscosity at $25^{\circ} \mathrm{C}$; Data from the CRC Handbook of Chemistry and Physics. ${ }^{15} \alpha$ : experimental static polarisability ${ }^{16}$ (except for water ${ }^{17}$ ); H-bonding: hydrogen-bonded solvent structure. ${ }^{18}$

${ }^{\mathrm{b}}$ Chernyak. ${ }^{19}$

${ }^{\mathrm{c}}$ Barthel, Neueder, and Roch. ${ }^{20}$ 
TABLE II. Molar masses, radii, and volumes of ions and solvents. Ionic $a b$ initio radii $r_{\mathrm{i}}$ from Parsons and Ninham, ${ }^{22}$ ionic volumes $V_{\mathrm{i}}$ calculated as $4 / 3 \pi r_{\mathrm{I}}^{3}$; van der Waals volumes $V_{\mathrm{v} d \mathrm{w}}$ of solvent molecules calculated using the "geometrical descriptors" plugin from the MarvinSketch software, ${ }^{23}$ radii $r_{\mathrm{v} d \mathrm{w}}$ calculated by the authors from $V_{\mathrm{v} d \mathrm{w}}$.

\begin{tabular}{lccc}
\hline \hline Anion & $M\left(\mathrm{~g} \mathrm{~mol}^{-1}\right)$ & $r_{\mathrm{I}}(\AA)$ & $V_{\mathrm{I}}\left(\AA^{3}\right)$ \\
\hline $\mathrm{Na}^{+}$ & 22.99 & 0.61 & 0.95 \\
\hline $\mathrm{F}^{-}$ & 19 & 1.02 & 4.4 \\
$\mathrm{Cl}^{-}$ & 35.45 & 1.69 & 20.2 \\
$\mathrm{Br}^{-}$ & 79.9 & 1.97 & 32.0 \\
$\mathrm{I}^{-}$ & 126.9 & 2.12 & 39.9 \\
$\mathrm{ClO}_{4}^{-}$ & 99.45 & 2.17 & 42.8 \\
$\mathrm{SCN}^{-}$ & 58.08 & 2.18 & 43.4 \\
$\mathrm{OAc}$ & & 2.2 & 44.6 \\
\hline $\mathrm{Solvent}^{-}$ & 59.04 & $r_{\mathrm{vdw}}(\AA)$ & $V_{\mathrm{vdw}}\left(\AA^{3}\right)$ \\
\hline Water & $M(\mathrm{~g} \mathrm{~mol}$ & \\
$\mathrm{MeOH}$ & 18.01 & 1.8 & 24.24 \\
$\mathrm{FA}$ & 32.04 & 2.1 & 36.84 \\
DMSO & 45.04 & 2.1 & 41.7 \\
$\mathrm{PC}$ & 78.13 & 2.6 & 72.69 \\
\hline \hline
\end{tabular}

sphere of hydration tightly bound, resulting in a larger hydrodynamic volume and a shorter retention time than expected. On the other hand, larger polarisable ions such as bromide, iodide, or thiocyanate are capable of directly interacting with the stationary phase, behaving as "sticky" ions. This latter finding was also demonstrated by Washabaugh and Collins by showing the temperature-dependence of their retention times.

Washabaugh and Collins also introduced the classification of ions into "kosmotropes" ("that turn towards order," as referred to their ordering of the surrounding water molecules) and "chaotropes" (thought to disorder the surrounding water molecules), based on their chromatographic behaviour. The anions that elute before $\mathrm{Cl}^{-}$on a Sephadex G-10 column are kosmotropes, whereas the ones that elute after $\mathrm{Cl}^{-}$are chaotropes. This terminology quickly became popular in several fields regarding electrolytes (the term chaotrope was already in use and had been introduced by Hamaguchi and Geiduschek, ${ }^{24}$ but Washabaugh and Collins coined "kosmotrope" to describe the ions that behave in the opposite way). These terms have become almost synonymous with the phrases "structure-maker" and "structure breaker" that have been used since the 1950s, but "kosmotrope" and "chaotrope" do not imply a long-range effect on the structure of water, whereas the former terms do. We note that the use of all these terms is currently undergoing revision.

These experiments contributed to the formulation of the "Law of Matching Water Affinities" (LMWA) ${ }^{25-27}$ and also provided evidence for the importance of dispersion forces on the behaviour of ions in water. ${ }^{28-31}$ The promise that size exclusion chromatography could generate comparably useful information for ions in non-aqueous solvents directed us to adapt this experiment for use with non-aqueous solvents.

An important area of ion specificity is the interaction of electrolytes with polymers and proteins. The second experiment we describe relates to the conformational behaviour of charged polymeric brushes in the presence of electrolytes. It is known that in aqueous systems the counter-ion of the brush strongly influences the conformation of the brush. ${ }^{32,33}$ Azzaroni et al. ${ }^{34}$ studied the ion-specific properties and behaviour of poly(2-methacryloyloxyethyl-trimethylammonium chloride) (PMETAC) brushes in water. They showed, by coupling QCM with atomic force microscopy, infrared spectroscopy, and contact angle measurements, that PMETAC brushes in water undergo total collapse in the presence of $\mathrm{ClO}_{4}{ }^{-}$. The response of PMETAC brushes in water to Hofmeister anions and cations has been characterised in detail by Kou et al. ${ }^{35}$ who found that the Hofmeister anions interact with the charged sites on the brush to different degrees, in agreement with the law of matching water affinities which is consistent with the expectations based on dispersion interactions.

\section{MATERIALS AND METHODS}

\section{A. Size exclusion chromatography}

Size exclusion chromatography (SEC) is a form of liquid chromatography. It is the only type of chromatography where no interaction is sought between the analyte and the stationary phase. The column therefore acts merely as a sieve. The separation or characterisation of a substance of a particular solvodynamic radius (which includes the size of the molecule or ion and any tightly bound solvent) is therefore obtained on a purely entropic basis. Separation is based on differences in the average path an analyte traverses inside the column. This is correlated to the size of the analyte with respect to the porosity of the stationary phase. More accurately, the analyte partitions between the moving eluent phase outside the pore space and the pools of stationary eluent inside the pores. ${ }^{36}$ If the molecule is sufficiently large that it is excluded from accessing the pores of the stationary phase, its path will be short and it will exit the column together with the eluent front. Alternately, if the analyte can access the pores, there will be a fraction of its molecules inside the pores at any time; therefore, its elution volume will be greater, the smaller the size. The longest paths are available when the solute can access all the porosity. In practical terms, this is when the solute is as small as the molecules of solvent.

The eluted analyte is characterised by its retention factor $K_{\text {SEC }}{ }^{36,37}$

$$
K_{\mathrm{SEC}}=\left(V_{\mathrm{R}}-V_{0}\right) / V_{\mathrm{i}},
$$

where $V_{\mathrm{R}}$ is the retention volume of the analyte peak, $V_{0}$ is the void volume of the column (the volume of the interstitial mobile phase), and $V_{\mathrm{i}}$ is the internal pore volume of the column (the stagnant volume of solvent trapped inside the stationary phase pores). A $K_{\mathrm{SEC}}=0$ indicates that the analyte is completely excluded from accessing the stationary phase pores, whereas $K_{\mathrm{SEC}}=1$ indicates that the analyte accesses all volumes accessible to the solvent. Molecules with intermediate sizes will display a retention factor between 0 and 1 , proportional to the size. A $K_{\mathrm{SEC}}>1$ is indicative of specific absorption to the stationary phase packing and is a non-ideal condition that violates the assumption of exclusively entropic separation. Experimentally, the void volume $V_{0}$ is measured by running a sample that is larger than the cut-off porosity of the stationary phase. A common choice for determining $V_{0}$ 
is Blue Dextran. This is what we used where possible. The internal pore volume $V_{\mathrm{i}}$ can be measured by subtracting $V_{0}$ from the elution volume of a molecule of similar size to the eluent. As the detection of such a molecule is often difficult, a molecule larger than the eluent but much smaller than the analyte is used, or the total geometric volume $V_{\mathrm{t}}$ of the column is used as an estimate of the internal volume. This is simply the volume of the cylinder with the same height and radius of the stationary phase of the column. In this second case, $K_{\mathrm{SEC}}$ is approximated as $K_{\text {av }}$ (available), where

$$
K_{\text {av }}=\left(V_{\mathrm{R}}-V_{0}\right) /\left(V_{\mathrm{t}}-V_{0}\right) .
$$

As this estimate does not account for the volume occupied by the packing material and the molecules of solvent tightly bound to the packing, this method results in an underestimation of the real retention factor of the analyte.

\section{B. Chromatography experiments}

The detailed description of our experimental arrangement and procedure is provided in Sec. SIII of the supplementary material. The chromatographic arrangement and experimental procedures used by Washabaugh and Collins ${ }^{21}$ were followed where possible, although substantial differences could not be avoided in our experiments, and they are summarised in Sec. SIII.A of the supplementary material. The usage of nonaqueous solvents presents a number of challenges including the measurement of the void volume and internal volume. A detailed description of the methods we have used to determine these volumes are included in Sec. SIII.F of the supplementary material.

\section{Quartz crystal microbalance}

A QCM (Quartz Crystal Microbalance) employs a quartz crystal resonator to detect mechanical and rheological changes. For experiments in solution, this could be a change in the solvent, adsorption or desorption of material from the sensor surface, or a change in conformation of molecules on the sensor surface. Commonly, the resonant frequency $\Delta f_{n}$ and the energy dissipation $\Delta D_{n}$, which characterises the free decay of the sensor oscillation, are measured. Here, $n$ is the order of the overtone. We typically use the third overtone, as the first overtone is susceptible to drift associated with the mounting of the resonator. When measuring liquids, the mass sensed by the resonator is not just the mass of the film on the surface, but also includes the mass of any solvent that is coupled to the resonator. ${ }^{38}$ In systems where a polymer film is covalently bound to the sensor, the mass of the film cannot change, but the coupled mass can change in response to the configuration of the polymer and/or changes in the solvent.

A Biolin Scientific Q-sense QCM-D was used to measure the $\Delta f_{3}$ and $\Delta D_{3}$ of the sensor in the presence of $10^{-3} \mathrm{~mol} \mathrm{~kg}^{-1}$ electrolyte solutions. For the measurements described here, the sensors have been functionalised with a polymer brush of poly(2-methacryloyloxyethyl-trimethylammonium chloride) (PMETAC); see Fig. S3 in the supplementary material for the chemical structure. As the mass of the film does not change, the observed change in $\Delta f_{n}$ reflects a change in the amount of coupled solvent, which accompanies conformational changes in the polymer film. Swelling of the film leads to an increase in coupled mass and a corresponding decrease in $\Delta f_{n}$. A swollen film is more viscous and therefore an increase in $\Delta D_{n}$ reflects swelling of the film.

As the viscosity and density of an electrolyte solution vary depending on the concentration and type of electrolyte, the resonant frequency and dissipation of the quartz resonator will be affected by changes in the solution. As a result, the measured $\Delta f$ and $\Delta D$ relative to the neat solvent include both the signal of interest (changes in brush conformation in response to electrolyte) and the response to the altered properties of the solution. The effect of $\mathrm{NaBr}$ concentration on the blank sensor in different solvents is shown in Fig. S4 of the supplementary material. In order to isolate the response of the film, the $\Delta f_{n}$ and $\Delta D_{n}$ caused by the same solution on a blank sensor were recorded and subtracted from the shifts measured in the presence of the polymer brush. This correction method is simplistic as it assumes the effects to be additive, but it is a simple and accessible way of estimating the change in sensor environment. Figure S7 in the supplementary material shows both the brush-coated sensor and bare sensor shifts and demonstrates a clear distinction between the two responses.

The gold-coated resonators used in the QCM-D were cleaned by piranha solution (volume fractions $\varphi_{\mathrm{H}_{2} \mathrm{O}_{2}}=0.3$, $\left.\varphi_{\mathrm{H}_{2} \mathrm{SO}_{4}}=0.7\right)$ for $10 \mathrm{~min}$ at $60{ }^{\circ} \mathrm{C}$, then rinsed with copious amounts of ultra-pure water, and dried under a nitrogen stream before use. The clean resonators were immersed in a $5 \times 10^{-3} \mathrm{~mol} \mathrm{dm}^{-3}$ solution of MUBB ( $\omega$-mercaptoundecyl bromoisobutyrate) in anhydrous ethanol for $\approx 24 \mathrm{~h}$ at room temperature to form a uniform monolayer of initiator with a thickness of $\approx 1.2 \mathrm{~nm}$, as determined by ellipsometry. PMETAC brushes were prepared using the SI-ATRP (surface initiatedatom transfer radical polymerisation) method. ${ }^{39}$ Typically, METAC (2-methacryloyloxyethyl-trimethylammonium chloride) $\left(5.94 \mathrm{~g}, 2.86 \times 10^{-2} \mathrm{~mol}\right)$, the free initiator 2 -EBiB (ethyl 2-bromoisobutyrate) $\left(2.7 \times 10^{-2} \mathrm{~g}, 1.4 \times 10^{-4} \mathrm{~mol}\right)$, and BPy (2,2-dipyridyl) $\left(4.4 \times 10^{-2} \mathrm{~g}, 2.8 \times 10^{-4} \mathrm{~mol}\right)$ were dissolved in $20 \mathrm{ml}$ of methanol/water mixture $\left(\varphi_{\mathrm{MeOH}}=0.8\right.$, $\left.\varphi_{\mathrm{H}_{2} \mathrm{O}}=0.2\right)$. Then, the initiator-modified substrates were placed inside the flask, and the solution was stirred at $25^{\circ} \mathrm{C}$ under argon for $120 \mathrm{~min}$ to remove dissolved oxygen. Afterward, $\mathrm{CuBr}\left(2 \times 10^{-2} \mathrm{~g}, 1.4 \times 10^{-4} \mathrm{~mol}\right)$ and $\mathrm{CuBr}_{2}\left(3 \times 10^{-3} \mathrm{~g}\right.$, $\left.1.4 \times 10^{-5} \mathrm{~mol}\right)$ were quickly added under argon protection at $25{ }^{\circ} \mathrm{C}$. The polymerization was allowed to proceed for a specific time to prepare the PMETAC brushes. At the end of polymerization, the resonators grafted with PMETAC brushes were washed with water and methanol and then soaked in a methanol/water mixture $\left(\varphi_{\mathrm{MeOH}}=0.8, \varphi_{\mathrm{H}_{2} \mathrm{O}}=0.2\right)$ overnight to remove the ligand and unreacted monomer.

Details about the experimental procedure are provided in Sec. SIV of the supplementary material.

\section{Experimental details}

The salts and the solvents were used as received apart from the drying of the salts. Anhydrous materials were used for the final runs of all experiments, whereas preliminary runs were performed with non-anhydrous solvents. Detailed information about the specifications of the materials used and the procedures employed are provided in Secs. SI and SII of the supplementary material. 
TABLE III. Summary of the electrolytes investigated per solvent.

\begin{tabular}{lcccc}
\hline \hline Electrolyte & MeOH & FA & DMSO & PC \\
\hline $\mathrm{NaOAc}$ & $\checkmark$ & $\checkmark$ & & \\
$\mathrm{NaF}$ & $\checkmark$ & & & \\
$\mathrm{NaCl}$ & $\checkmark$ & $\checkmark$ & $\checkmark$ & $\checkmark$ \\
$\mathrm{NaBr}$ & $\checkmark$ & $\checkmark$ & $\checkmark$ & $\checkmark$ \\
$\mathrm{NaI}$ & $\checkmark$ & $\checkmark$ & $\checkmark$ & $\checkmark$ \\
$\mathrm{NaClO}_{4}$ & $\checkmark$ & $\checkmark$ & $\checkmark$ & $\checkmark$ \\
$\mathrm{NaSCN}^{\mathrm{NaSCN}}$ & $\checkmark$ & $\checkmark$ & $\checkmark$ & \\
\hline \hline
\end{tabular}

A salt concentration that was accessible for most electrolytes in the different solvents is $0.05 \mathrm{~mol} \mathrm{~kg}-1$. Therefore this was set as the electrolyte concentration for the chromatography experiments. QCM measurements were performed with electrolyte solutions of $10^{-3} \mathrm{~mol} \mathrm{~kg}{ }^{-1}$ concentration. This is because this lower concentration was sufficient to produce detectable changes in the polymeric brush behaviour, whilst higher concentrations had little effect on the magnitude of the changes observed (see Fig. S5 in the supplementary material). Table III summarises the electrolytes investigated for each solvent. Although there are literature reports ${ }^{40}$ that $\mathrm{NaBr}$ is soluble in propylene carbonate at a concentration of $5 \times 10^{-2} \mathrm{~mol} \mathrm{~kg}^{-1}$, it was not possible to achieve its solubilisation, neither at that concentration nor at the lesser concentration of $10^{-3} \mathrm{~mol} \mathrm{~kg}^{-1}$. Note the solubility of salts varies greatly across solvents and is in most cases lower than in water (see Table S4 in the supplementary material). This limits the range of combinations and concentrations available for investigation.

In order to avoid unwanted contamination from the dissolution of materials in the non-aqueous solvents employed, polymers and elastomers with excellent chemical resistance were used in all the experiments. These include Saint-Gobain Tygon ${ }^{\circledR} 2001$ for the QCM peristaltic pump tubing, DuPont ${ }^{\mathrm{TM}}$ Karlez ${ }^{\circledR} 6380$ for the QCM O-ring and internal cell gasket, Teflon® tubing for the QCM sample cell connections, and polytetrafluoroethylene (PTFE) and fluorinated ethylene propylene (FEP) tubing for the SEC experiments.

\section{RESULTS}

\section{A. Chromatography}

Chromatograms were measured for a range of electrolytes in water, methanol, formamide, dimethyl sulfoxide, and propylene carbonate in order to investigate the influence of solvent on the ion specificity of elution. For all of the chromatograms, the baseline conductivity of the pure solvent has been subtracted and the values of the conductivity are rescaled relative to the maximum conductivity being set to 1 . Figure 1 illustrates the chromatograms in the different solvents. Based on the sizes of the ions (see Table II), the ordering of elution expected in ideal conditions is $\mathrm{CH}_{3} \mathrm{COO}^{-}<\mathrm{SCN}^{-}<\mathrm{ClO}_{4}^{-}<\mathrm{I}^{-}<\mathrm{Br}^{-}$ $<\mathrm{Cl}^{-}<\mathrm{F}^{-}$, noting that the shapes of the polyatomic ions thiocyanate, perchlorate, and acetate are expected to have an influence on their solvodynamic radius and therefore may influence their positioning.
Water is the only solvent in which we have measured the internal volume accurately in addition to the void volume. For all of the non-aqueous solvents, only the geometric volume of the packing has been measured and the internal volume has been estimated by using the ratio of internal volume to geometric volume found for water-noting that the swelling of the column is solvent dependent. Such an estimate of the internal volume is not precise due to the different levels of swelling and different molar volumes of the different solvents. However, we think it is still a better estimate than the geometric volume, particularly for interpreting the elution of electrolytes. Therefore, we have used the estimate of the internal volume for the calculation of $K_{\text {SEC }}$. Regardless, our primary interest is in the ion-specific trends in each solvent and these are not impacted by the calculation of $K_{\mathrm{SEC}}$. The (measured) geometric volume and the calculated internal ones are indicated in each of the non-aqueous chromatograms. We see that the elution order in water [Fig. 1(A)] follows the Hofmeister series with the exception that the order of $\mathrm{SCN}^{-}$and $\mathrm{ClO}_{4}{ }^{-}$ is inverted. This is in agreement with the earlier experiments of Washabaugh and Collins. Notably, ordering of the anions does not follow the "naked" size of the anions, but rather the reverse trend is observed. This is consistent with the small ions being strongly hydrated and the larger polarisable ions being strongly attracted to the stationary phase and only eluting after a full column volume of water has passed. That is, retention in the column is observed for the strongly chaotropic anions. Acetate, which is a large ion but has a charged group of low static polarisability, elutes where expected based on its size, therefore ideally. Whereas fluoride, which is substantially smaller than acetate, elutes before it. This indicates a very large and strongly bound hydration sphere for fluoride. Notably the shape of the acetate elution peak exhibits a shoulder and in this respect it differs from the other peaks which are symmetrical. This may be due to some of the acetate being present as the undissociated acid.

The elution order in methanol [Fig. 1(B)] follows precisely the same order as water. The overall elution volumes are much greater, and all of the electrolytes required an elution volume greater than the internal volume, suggesting that all of the ions except acetate and fluoride interact with the column, i.e., are "sticky," but the degree with which they interact with the column remains size dependent. For both water and methanol, the observation is that the smaller the ion, the weaker the interaction with the column.

When formamide is the solvent, the chromatogram changes dramatically [Fig. 1(C)]. The elution volumes for all ions are very similar, indicating much weaker ion specificity. Formamide is the eluent in which the stationary phase has the largest porosity (see Table S5 in the supplementary material). This could be offered as an explanation for the lower selectivity, but the expectation is that the pore sizes are still far larger than the ion sizes. The precise elution order is difficult to quantify because of the small differences in the elution times and the presence of double peaks for $\mathrm{Cl}^{-}$and $\mathrm{F}^{-}$. We do not understand the presence of the double peaks, although in SEC the fronting, tailing, and splitting of peaks can also be induced by reversible association of the analyte in solution, such as in the formation of dimers. Theoretical studies on protein 

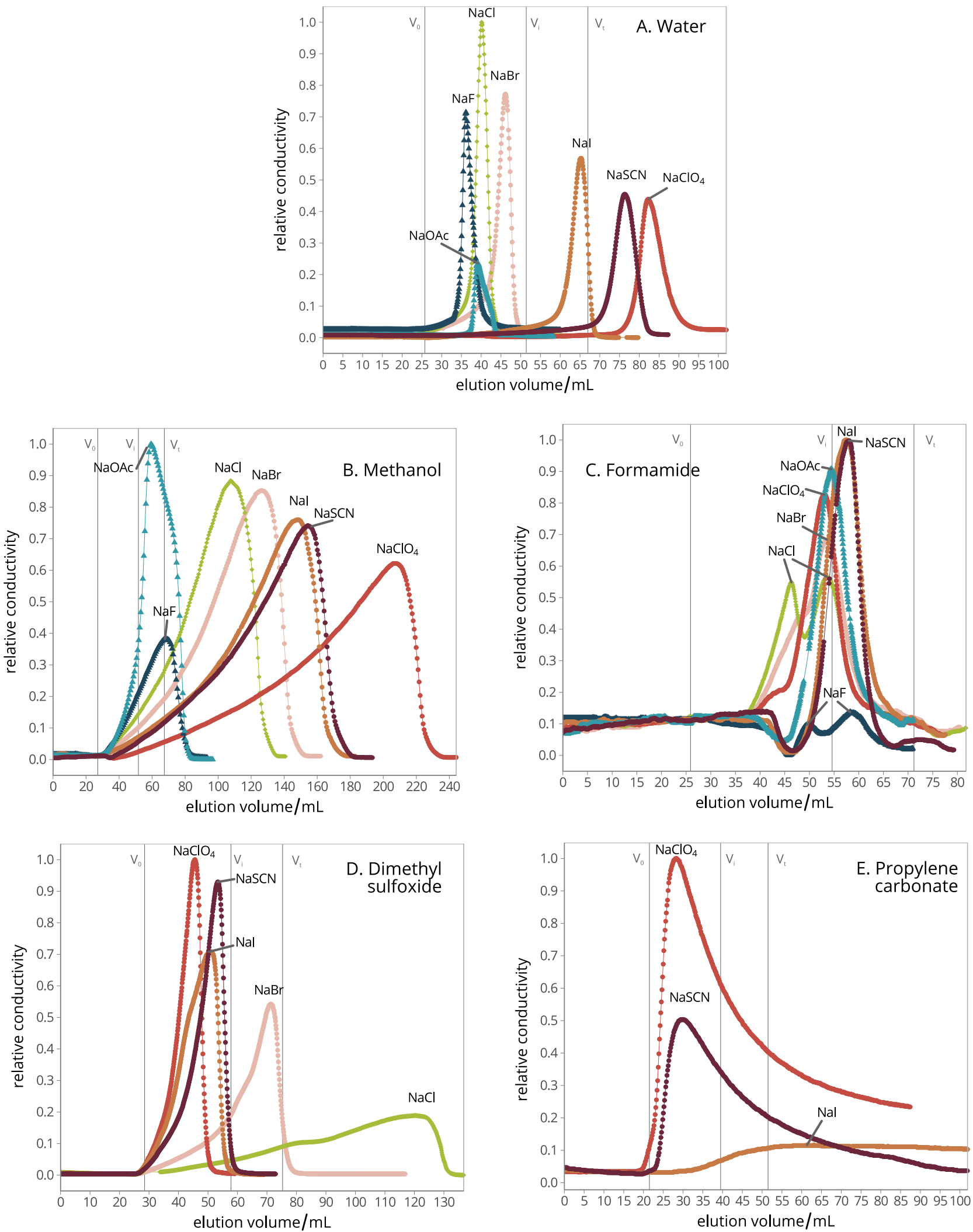

FIG. 1. Size exclusion chromatograms for a range of electrolytes in water (A), methanol (B), formamide (C), dimethyl sulfoxide (D), and propylene carbonate (E). The conductivity of the pure solvent has been subtracted and the signal has been normalised to the highest peak such that the relative conductivity ranges between 0 and 1 and is dimensionless. The elution volume is expressed in millilitres of solvent and is compared to the void volume, $V_{0}$, the internal volume $V_{\mathrm{i}}$, and the geometric volume $V_{\mathrm{t}}$.

association in SEC predict that, when the association and dissociation rates are comparable to the eluent convection rate, a merged, broad peak forms, with either fronting or tailing when dimers or monomers dominate, respectively. ${ }^{41}$ When both the association and dissociation kinetics are slow, split peaks form. For ions in solution, the associated species have to be 
identified. It is possible that they are contact or solvent-shared ion pairs in solution. ${ }^{41}$

The chromatogram for DMSO [Fig. 1(D)] shows the reversal of the elution pattern observed in protic solvents. As such, the order observed is that expected based on the size of the anion alone. Whilst the order suggests that size is controlling the elution, long elution times for chloride and bromide indicate that they are interacting strongly with the stationary phase. These sticky ions are now kosmotropic anions in contrast to water and methanol where the chaotropic ions presented as sticky. In this case the smaller less polarisable ions have a greater affinity for the stationary phase.

The range of electrolytes that could be studied in propylene carbonate is limited [Fig. 1(E)]. The available data suggests similar behaviour to that observed in DMSO. However, in this case, iodide behaves as strongly sticky.

The elution behaviour for the various electrolytes are summarised in Fig. 2. Here the value of $K_{\mathrm{SEC}}$ is shown for the various electrolytes (ordered from top to bottom as the Hofmeister series, i.e., the fundamental series for anions) in the different solvents. When presented in this manner, if $K_{\mathrm{SEC}}$ values increase when going down the series, a Hofmeister series is evident. If the $K_{\mathrm{SEC}}$ values decrease when going down the series, a reverse Hofmeister series is evident. It is apparent that the ion specificity in the SEC for water and methanol follows the Hofmeister series, whereas the reverse Hofmeister series is observed for PC and DMSO. The $K_{\mathrm{SEC}}$ values for formamide vary little, as no such series is evident.

Overall, we see that water and methanol behave very differently to DMSO and PC. One might therefore ascribe the difference to the protic and aprotic nature of the solvents, respectively. However, the protic solvent formamide appears to present an intermediate case, in which case, the binary interpretation of protic versus aprotic is not sensible. Rather, the effects of the solvents may be due to their polarisabilitynoting that the aprotic solvents studied here are larger and more polarisable. The overall picture that emerges is that in solvents of low static polarisability (water and methanol, see Table I), the polarisable ions interact strongly with the stationary phase, whereas in solvents of higher static polarisability (DMSO and PC) the more polarisable ions preferentially interact with the solvent - and therefore do not interact strongly with the stationary phase. Formamide, which has a static polarisability greater than that of water and methanol but less than that of DMSO and PC, presents an intermediate situation in which the ion specificity is weak and the ordering is unclear.

\section{B. QCM}

The monitoring by QCM of the conformation of a brush grafted to a quartz crystal resonator can be interpreted as follows: if the oscillation frequency increases (loss in coupled mass) and the dissipation decreases (coupled mass behaving as more elastic and less viscous), the brush is collapsing. If the opposite is observed, the brush is swelling with respect to its status in the neat solvent.

The results of the QCM measurements are summarised in Fig. 3. Here $\Delta F_{3}$ (the normalised frequency shift $\Delta f_{3} / 3$ ) and $\Delta D_{3}$ provide for a consistent picture of how ions influence the conformation of the PMETAC polymer film in different solvents. The ions are ordered according to the fundamental series for anions (i.e., the Hofmeister series). It is clear that the protic solvents, water, methanol, and formamide, all exhibit specific-ion effects that follow the Hofmeister series. The series is reversed in the aprotic solvents DMSO and PC.

In formamide and DMSO, the effects of the electrolytes are smaller compared to the other solvents. The PMETAC polymer film carries a positive charge through the dissociation of the chloride cation from the ammonium groups. These charged groups promote the swelling of the film. Therefore a collapse of the film is associated with a decrease in the overall positive charge due to incorporation of anions into the film. Conversely, swelling of the film infers an increase in the positive charge or osmotic pressure in the film associated with ingress of kosmotropic anions into the brush. The latter requires that the kosmotropic anions do not form contact ion pairs with the ammonium groups of the brush. ${ }^{42}$

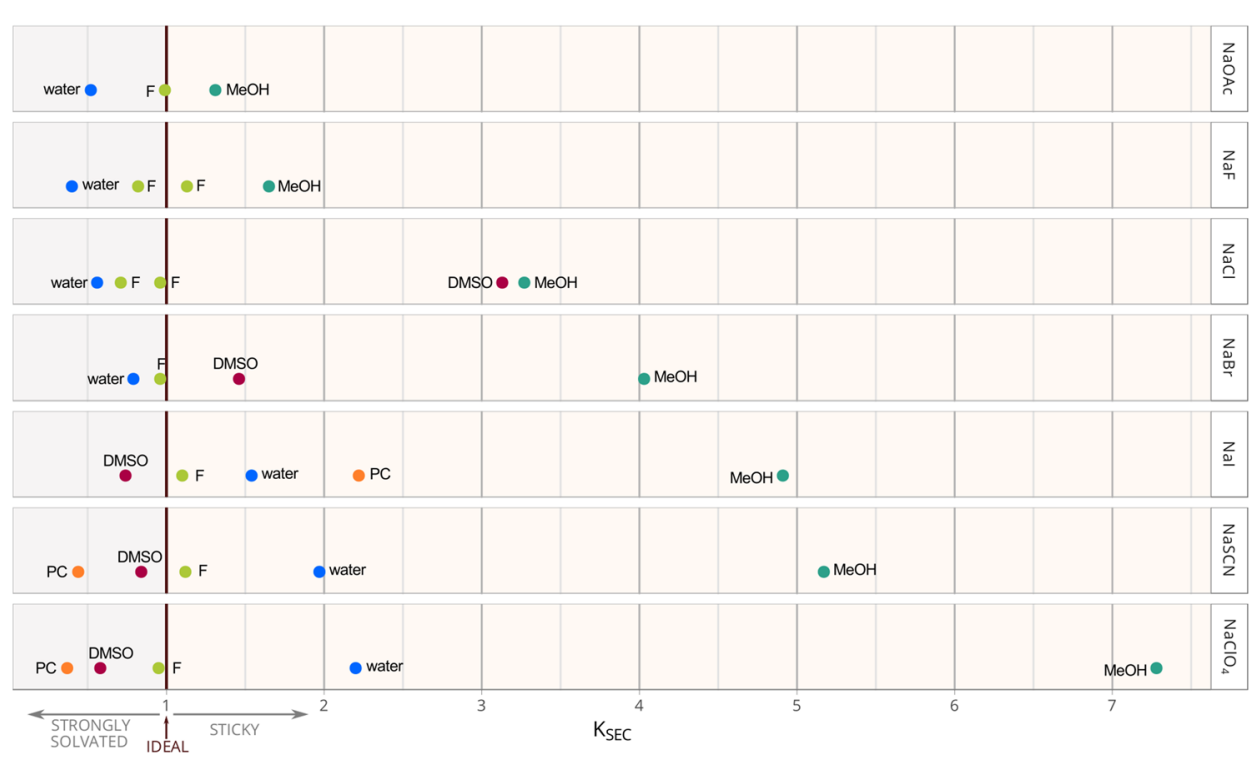

FIG. 2. Summary of the retention factors, $K_{\mathrm{SEC}}$, of each electrolyte when eluted with different solvents. The electrolytes are listed from top to bottom according to the Hofmeister series (kosmotropic to chaotropic). The labels on the bottom left indicate the thresholds characterising different behaviours of the ions in solution. This plot elucidates the $K_{\mathrm{SEC}}$ trends in different solvents: methanol and water show the same ordering, formamide shows no selectivity, PC and DMSO show an ordering that is opposite to methanol and water. 

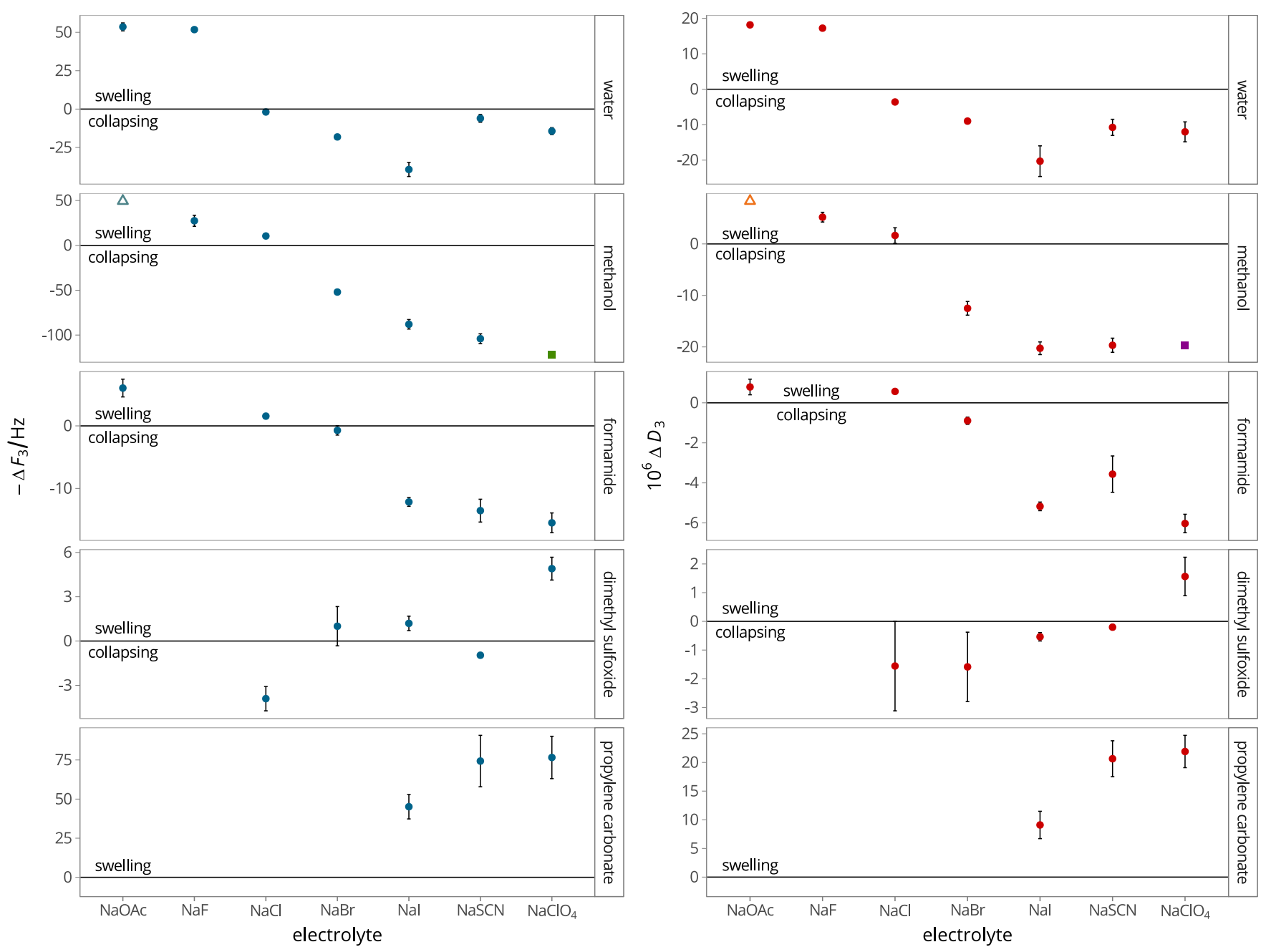

FIG. 3. Summary of frequency and dissipation shifts produced by a $10^{-3} \mathrm{~mol} \mathrm{~kg}^{-1}$ electrolyte solution on a PMETAC brush in different solvents. The corresponding conformational change of the brush is indicated on the plots. In $\mathrm{MeOH}$, the electrolytes $\mathrm{NaOAc}$ and $\mathrm{NaClO}_{4}$ have not been measured in anhydrous conditions. For $\mathrm{NaOAc}$, the value from "as supplied" $\mathrm{MeOH}$ has been used and is represented by the empty triangle symbol. $\mathrm{NaClO}_{4}$ has not been tested in dry conditions as the brush is difficult to re-swell afterwards, as seen by our non-anhydrous tests. The value is an estimate calculated from the non-anhydrous $\mathrm{NaClO}_{4}$ measurement by using the ratio of the anhydrous to non-anhydrous response of NaSCN and is indicated by a square symbol. $\Delta F_{3}$ is the normalised frequency shift $\Delta f_{3} / 3$.

Figure $\mathrm{S} 6$ in the supplementary material shows the response of the PMETAC brush to $\mathrm{NaClO}_{4}$ solutions in the different solvents. The brush displays the most extremal behaviour for this electrolyte in the different solvents. The same effect has been observed previously for water. ${ }^{34,35}$

In order to investigate changes in the viscoelastic properties of the brush, a plot of $\Delta D_{3}$ versus $-\Delta F_{3}$ can be constructed for all the electrolytes in the different solvents (see Fig. S8 in the supplementary material). We note that to a good approximation these form a line of constant slope, indicating that the electrolytes do not substantially alter the viscoelasticity of the brush.

\section{DISCUSSION}

We have described studies of specific-anion effects in two different systems using the same range of solvents. Our interest is not primarily in the results of these individual experiments, but rather in observing the trends observed in each experiment and comparing them to the fundamental series- the observed ion-specific trend at infinite dilution in the absence of surfaces-in order to evaluate how the fundamental series is perturbed in the presence of significant concentrations of electrolytes and in the presence of surfaces. The nature of the chromatography and QCM experiments are very different. In the chromatography experiments, we have a polar largely uncharged stationary phase whereas in the QCM experiments we have a polymer brush terminated in cationic amine groups. Despite the differences between these experiments, the trends in the specific-ion effects are consistent. Taking a dichotomic view, we can say that in the protic solvents the fundamental series is observed (Hofmeister) whereas the series is reversed in the aprotic solvents. However, this binary interpretation is an oversimplification. In the QCM experiments, the trend in formamide followed the results obtained in water and methanol, whereas in the SEC experiments no clear trends were observed in formamide. The aprotic solvents are considerably more polarisable than the protic solvents, with formamide being the most polarisable protic solvent. The results we observed can be interpreted as resulting in a competition between the solvent 
and the surface for the anion. The evidence suggests that in protic solvents the more polarisable ions interact more strongly with the surface (and the less polarisable ions do not interact strongly with the surface), whereas in aprotic solvents the more polarisable ions do not interact with the surface as they are more favourably solvated. This suggests that the trends observed are not due to the binary categorisation of protic and aprotic but rather reflect changes in the polarisability of the solvent. These findings show that a dichotomic view of the behaviour in protic versus aprotic solvent is oversimplified. Rather there is a gradation of behaviours, and the surface properties (both of the charged surface groups and the non-charged sites) determine where the "watershed" sits for the reversal of the observed trend. In the two investigations that we have performed, we can see that it is at formamide when Dextran is the surface, whereas it is at DMSO when PMETAC is the interacting surface (also note that the effects in formamide are also small-whilst following the Hofmeister series in the QCM experiments).

We suggest that the observations can largely be explained by an evaluation of the dispersion forces between the ions and the solvent and the ions and the surface. Noting that these interactions will vary depending on the nature of the surface. This suggests that for a different class of surface (e.g., a metal oxide) the series may well be reversed. Thus we have the basis for which the huge complexity of specific-ion effects observed across different solvents and in the presence of different surfaces can arise. We have solvents of different polarisability competing for ions of different polarisability with surfaces that differ in polarisability and charge. This has the implication that, in order to model the specific-ion effects in different solvents, the polarisability of the solvent (and not just the dielectric permittivity) needs to be included, as the ion-solvent dispersion interactions play a fundamental role in specific-ion effects.

The trends we observe here lead us to propose a rule of thumb. We expect that specific-ion trends in weakly polarisable solvents follow those seen in water, whereas specific-ion trends in strongly polarisable solvents follow the reverse trend, whereas solvents of intermediate polarisability may not exhibit a clear trend. Given the paucity of data on specific-ion effects in non-aqueous solvents compared to aqueous solvents, this rule of thumb may provide a useful starting point for engineering specific-ion effects in non-aqueous solvents. As such we expect that the polarisability of the substrates is also important in affecting specific-ion effect trends and as such metal and metal oxide surfaces may well present different trends to those observed here. Furthermore, the studies reported here all employed $\mathrm{Na}^{+}$as the cation. We have chosen to vary the anion as the ion specificity of anions is often stronger than cations. It is known that the particular pairing of ions is important, as the specific-ion effects series can change and even reverse depending on the counterion. ${ }^{25,43-46}$ We have chosen to limit our studies to the sodium cation in order to reduce the parameter space to an acceptable level. Further studies examining the effect of the cation are warranted.

This has not been investigated here and remains a challenge for future investigations, with important consequences for some applications. For each experiment, only direct testing currently allows to determine how the counterion affects the specific-ion series ordering, as the variables at play are many, and the balancing of forces subtle. The present and future advancement of theories will of course achieve better predictive power. In addition, these studies also highlight the importance of investigating and identifying the products of ion association in solution.

\section{A. The influence of trace quantities of water}

When investigating specific-ion effects in polar nonaqueous solvents, which are to some extent hygroscopic, the possible interference of water is a concern. Water should not be present in solution in concentrations comparable with those of the ions that are investigated, and if possible, it should be oneorder of magnitude lower. ${ }^{47}$ It is known that water can selectively solvate ions in mixtures with non-aqueous solvents. ${ }^{48}$ Preferential solvation is found to be negligible for mixtures of solvents of similar polarity and/or hydrogen-bonding capability $^{49}$; therefore, we can expect trace concentrations of water in $\mathrm{MeOH}$ and FA to be distributed randomly in solution and within the solvation shells of the ions. In the case of solvents that differ in polarity or hydrogen-bonding properties to water, such as DMSO and PC, water preferentially solvates the anions, while the cations exhibit little to no preferential solvation. ${ }^{50}$ This matters particularly for the experiments in this work as they are probing specific-anion effects. Therefore some assessment of the amount of water in the solvation shell of the ions present is warranted.

Assuming that water distributes primarily in the solvation shell and assuming an hydration number of 6 , the concentration of water required to form a complete hydration shell around the ions present in a non-aqueous solvent can be calculated. We define the ratio $R=n_{\mathrm{H}_{2} \mathrm{O}} / n_{\mathrm{i}}$ as the number of water molecules per ion (counting both cations and anions). For electrolyte concentrations of $10^{-1} \mathrm{~mol} \mathrm{~kg}^{-1}, 5 \times 10^{-2} \mathrm{~mol} \mathrm{~kg}^{-1}$, and $10^{-3} \mathrm{~mol} \mathrm{~kg}^{-1}$, Table IV shows $R$ at different water concentrations $\mathrm{w}_{\mathrm{H}_{2} \mathrm{O}} \%$.

At the electrolyte concentration we used in the chromatography experiments $\left(5 \times 10^{-2} \mathrm{~mol} \mathrm{~kg}^{-1}\right) \mathrm{w}_{\mathrm{H}_{2} \mathrm{O}} \geq 1 \%$ is necessary in order for each salt ion (anion and cation) to be fully surrounded by a solvation shell of water molecules. The $\mathrm{w}_{\mathrm{H}_{2} \mathrm{O}} \%$ in our samples was always less than $0.1 \%$. This shows that for electrolytes at a concentration of $5 \times 10^{-2} \mathrm{~mol} \mathrm{~kg}^{-1}$ and above, the concentration of water is too low for water to dominate the solvation shell. For a water content of $0.1 \%$ by weight, which was the highest water content measured by Karl

TABLE IV. Water to ion ratio $R=n_{\mathrm{H}_{2} \mathrm{O}} / n_{\mathrm{i}}$ per $1 \mathrm{~kg}$ of solution calculated for the salt concentration indicated in parentheses (in $\mathrm{mol} \mathrm{kg}^{-1}$ ).

\begin{tabular}{lcllc}
\hline \hline $\mathrm{W}_{\mathrm{H}_{2} \mathrm{O}} \% \mathrm{a}$ & $\mathrm{mol}_{\mathrm{H}_{2} \mathrm{O} \mathrm{kg}}{ }_{\mathrm{s}}^{-1 \mathrm{~b}}$ & $\mathrm{R}(0.1)$ & $\mathrm{R}(0.05)$ & $\mathrm{R}(0.001)$ \\
\hline 0.01 & 0.006 & 0.03 & 0.06 & 3 \\
0.03 & 0.02 & 0.08 & 0.2 & 8 \\
0.10 & 0.06 & 0.3 & 0.6 & 28 \\
0.20 & 0.1 & 0.5 & 1 & 56 \\
0.35 & 0.2 & 1 & 2 & 98 \\
0.50 & 0.3 & 1.4 & 3 & 140 \\
1.00 & 0.6 & 3 & 6 & 281 \\
\hline \hline
\end{tabular}

${ }^{\mathrm{a}_{\mathrm{W}_{2} \mathrm{O}} \%}=m_{\mathrm{H}_{2} \mathrm{O}} / m_{\text {sample }} \times 100$.

b The subscript "s" stands for solvent. 

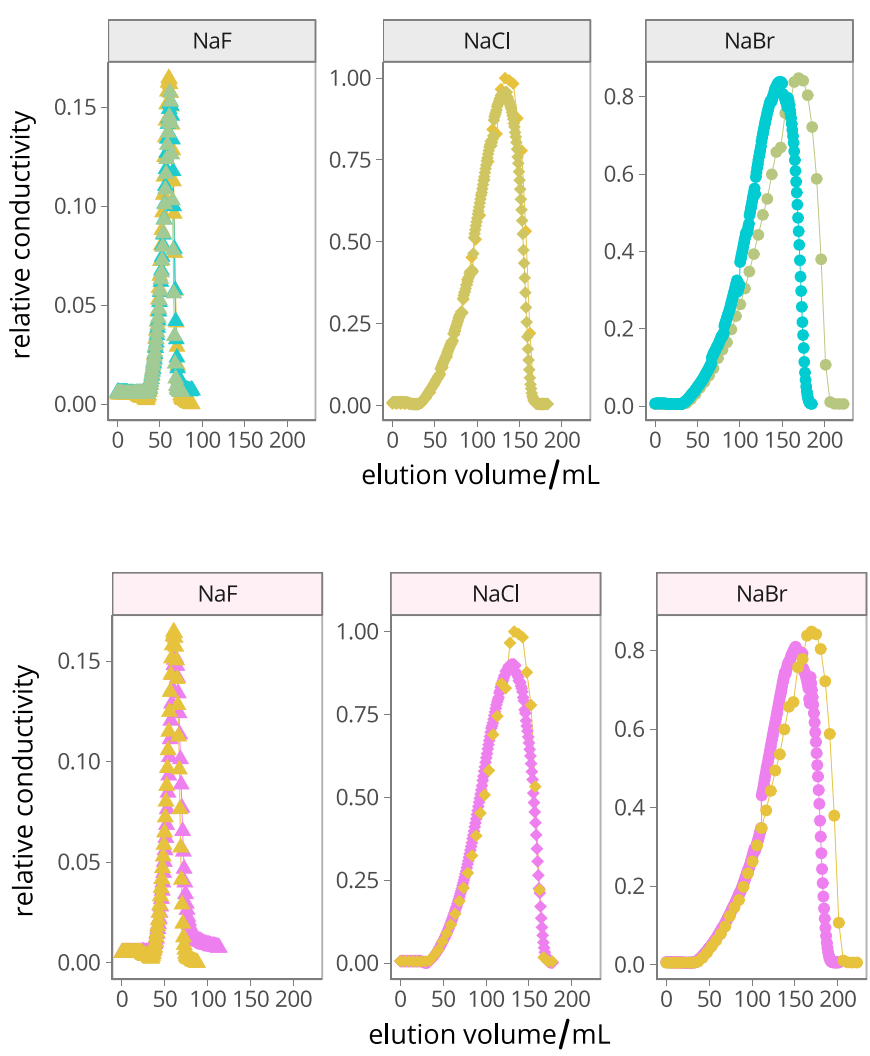

Fischer titration (Sec. SII of the supplementary material) in our "anhydrous" experiments, there is approximately 1 molecule of water for every 550 molecules of $\mathrm{MeOH}$. Similarly the ratio of solvent molecules to water molecules is 400 in FA, 230 in DMSO, and 175 in PC.

It is evident from Table IV that, as the electrolyte concentration decreases, lower contents of water are required to fully occupy the solvation shell of the ions present. This encourages the use of higher $\left(>5 \times 10^{-2} \mathrm{~mol} \mathrm{~kg}^{-1}\right)$ electrolyte concentrations, but the lower solubility of electrolytes in many nonaqueous solvents limits the available concentration. It is also not known whether being able to form a solvation shell around each ion would be enough for water to affect the specific-ion effects series in the non-aqueous system significantly, or if a higher amount of water than that is needed.

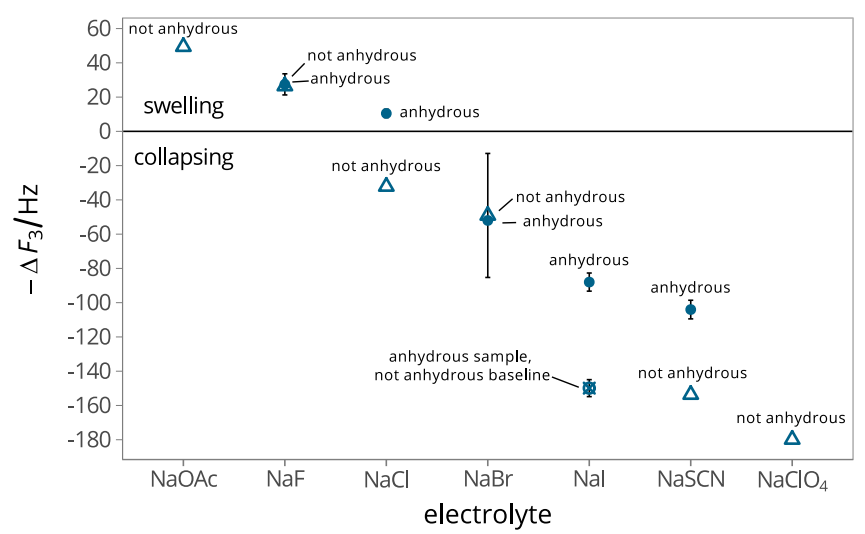

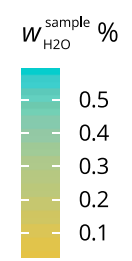

FIG. 4. Elution peaks of $\mathrm{NaF}, \mathrm{NaCl}, \mathrm{NaBr}$ samples with varying water concentrations in $\mathrm{MeOH}$. The baseline conductivity of the pure solvent has been subtracted and the values of the conductivity rescaled relative to the maximum conductivity being set to 1 .

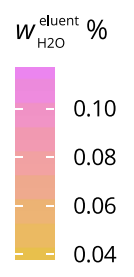

FIG. 5. Elution peaks of $\mathrm{NaF}, \mathrm{NaCl}, \mathrm{NaBr}$ in the presence of $\mathrm{MeOH}$ with differing water concentrations. The baseline conductivity of the pure solvent has been subtracted and the values of the conductivity rescaled relative to the maximum conductivity being set to 1 .
It is known, for instance, that the influence of water depends on it being available as a proton donor. Experiments show that traces of water are highly reactive with radical anions in acetonitrile, whereas the same content of water has almost no effects in DMF and DMSO. Therefore, in the latter two solvents water is solvated in a way that forbids it from acting as a proton donor: it is argued that this is because of hydrogen bonding. ${ }^{47}$

In order to address this, we have performed some experiments to determine the effect of the addition of small amounts of water on our experiments. In methanol, we have compared the chromatographic runs of $\mathrm{NaF}, \mathrm{NaCl}$, and $\mathrm{NaBr}$ when a small amount of water was added to either the sample or the eluent. Figures 4 and 5 show chromatograms in repeat runs when the water content in the sample or the eluent is

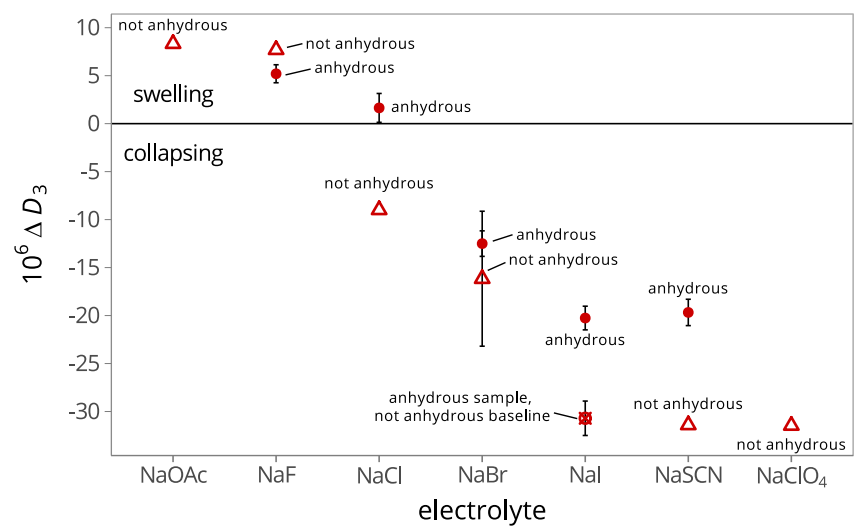

FIG. 6. Frequency and dissipation shifts obtained for dry versus non-dry methanol solutions. $\Delta F_{3}$ is the normalised frequency shift $\Delta f_{3} / 3$. 
approximately doubled. No significant effect is seen on the position of the peak after doubling the water content in the eluent or sample. This suggests that the results we observe are largely unaffected by the presence of small quantities of water.

Regarding the presence of water in the QCM experiments, we have to consider that the brush itself is hygroscopic. In addition, the electrolyte concentrations used is much lower, $10^{-3} \mathrm{~mol} \mathrm{~kg}^{-1}$; therefore, lower contents of water might be able to affect the system. A study of the shifts in anhydrous and non-anhydrous conditions has been performed for $\mathrm{MeOH}$ and is presented in Fig. 6. We observe that the presence of water in the solvent favours the ion-induced collapse of the brush for the chaotropic ions, whereas very little effect is seen for kosmotropic ions. Importantly, the presence of water does not appear to influence the observed trend in ion specificity.

Studies of the influence of water on the behaviour of non-aqueous systems are a topic that deserves thorough investigation. We hope that these observations can stimulate studies in the matter, and the results that have presented in the previous sections need to be considered in light of this information. The determination of how much water is important is unlikely to be a simple consideration. Rather it will depend on the information being sought. We think that it will be important to determine the concentration of water that leads to a measurable change as well as the concentration of water required to alter the observed ion specific trends.

\section{CONCLUSIONS}

We have experimentally investigated specific-ion effects in water and a range of non-aqueous solvents. We find that for the anions, specific-ion effects are exhibited in all solvents following the fundamental series (Hofmeister) or the reversal of the fundamental (reverse Hofmeister) series for the substrates investigated here. The key determinant in the solvent reversal of the series is the polarisability of the solvent. Weakly polarisable solvents give rise to trends in specific-ion effects akin to water, whereas strongly polarisable solvents give rise to the reverse trend. We argue that this is due to the relative strength of the dispersion interactions between the ion and the solvent and the ion and the surface. This for the first time provides a framework for understanding how ion-specific trends in non-aqueous solvents are related to ion-specific trends in water. That the latter will always be higher gives us more confidence that the trends observed here are not influenced by water.

\section{SUPPLEMENTARY MATERIAL}

See supplementary material for further details on four subjects. These are (1) materials and methods, (2) analysis of water by Karl Fischer titration, (3) details of the chromatography experiments, and (4) details of the QCM experiments.

\section{ACKNOWLEDGMENTS}

The authors thank Professor Pierandrea Lo Nostro and Dr. Andrea Salis for helpful discussion; Mr. Tim Sawkins and
Mr. Ron Cruikshank at the Department of Applied Mathematics, RSPE, ANU and the ANU RSPE Electronics Unit (Mr. David Anderson, Mr. Dennis Gibson, and Mr. Luke Materne) for technical support; Mrs. Avis Paterson, Ms. Bozena Belzowski, and Mr. Vance Lawrence at the ANU Research School of Chemistry for providing Blue Dextran. V.S.J.C. acknowledges the Australian Research Council for funding. G.M.L. acknowledges the financial support of the National Natural Science Foundation of China (Nos. 21574121 and 21622405).

${ }^{1}$ N. Schwierz, D. Horinek, and R. R. Netz, Langmuir 26, 7370 (2010).

${ }^{2}$ H. I. Okur, J. Hladílková, K. B. Rembert, Y. Cho, J. Heyda, J. Dzubiella, P. S. Cremer, and P. Jungwirth, J. Phys. Chem. B 121, 1997 (2017).

${ }^{3}$ T. T. Duignan, M. D. Baer, G. K. Schenter, and C. J. Mundy, Chem. Sci. 8, 6131 (2017)

${ }^{4}$ P. Lo Nostro and B. W. Ninham, Curr. Opin. Colloid Interface Sci. 23, A1 (2016).

${ }^{5}$ D. Bilaničová, A. Salis, B. W. Ninham, and M. Monduzzi, J. Phys. Chem. B 112, 12066 (2008).

${ }^{6}$ C. L. Henry and V. S. J. Craig, Langmuir 24, 7979 (2008).

${ }^{7}$ Z. Yang, J. Biotechnol. 144, 12 (2009).

${ }^{8}$ N. Peruzzi, B. W. Ninham, P. Lo Nostro, and P. Baglioni, J. Phys. Chem. B 116, 14398 (2012).

${ }^{9}$ N. Peruzzi, P. Lo Nostro, B. W. Ninham, and P. Baglioni, J. Solution Chem. 44, 1224 (2015).

${ }^{10}$ M. Giesecke, F. Hallberg, Y. Fang, P. Stilbs, and I. Furó, J. Phys. Chem. B 120, 10358 (2016).

${ }^{11}$ V. Mazzini and V. S. J. Craig, Curr. Opin. Colloid Interface Sci. 23, 82 (2016).

${ }^{12}$ V. Mazzini and V. S. J. Craig, Chem. Sci. 8, 7052 (2017).

${ }^{13}$ F. H. Arnold, Protein Eng. Des. Sel. 2, 21 (1988).

${ }^{14}$ A. S. Thomas and A. H. Elcock, J. Am. Chem. Soc. 129, 14887 (2007).

${ }^{15}$ CRC Handbook of Chemistry and Physics, 90th ed., edited by D. R. Lide (CRC Press/Taylor and Francis, 2010).

${ }^{16}$ R. Bosque and J. Sales, J. Chem. Inf. Comput. Sci. 42, 1154 (2002).

${ }^{17}$ L. Weiss, A. Tazibt, A. Tidu, and M. Aillerie, J. Chem. Phys. 136, 124201 (2012).

${ }^{18}$ H. D. B. Jenkins and Y. Marcus, Chem. Rev. 95, 2695 (1995).

${ }^{19}$ Y. Chernyak, J. Chem. Eng. Data 51, 416 (2006).

${ }^{20}$ J. Barthel, R. Neueder, and H. Roch, J. Chem. Eng. Data 45, 1007 (2000)

${ }^{21}$ M. W. Washabaugh and K. D. Collins, J. Biol. Chem. 261, 12477 (1986).

${ }^{22}$ D. F. Parsons and B. W. Ninham, J. Phys. Chem. A 113, 1141 (2009).

${ }^{23}$ ChemAxon, "Marvinsketch 17.27.0," https://www.chemaxon.com, 2017.

${ }^{24}$ K. Hamaguchi and E. P. Geiduschek, J. Am. Chem. Soc. 84, 1329 (1962).

${ }^{25}$ K. D. Collins, Biophys. J. 72, 65 (1997).

${ }^{26}$ K. D. Collins, Methods 34, 300 (2004).

${ }^{27}$ K. D. Collins, G. W. Neilson, and J. E. Enderby, Biophys. Chem. 128, 95 (2007).

${ }^{28}$ W. Kunz, L. Belloni, O. Bernard, and B. W. Ninham, J. Phys. Chem. B 108, 2398 (2004).

${ }^{29}$ D. F. Parsons, M. Boström, P. Lo Nostro, and B. W. Ninham, Phys. Chem. Chem. Phys. 13, 12352 (2011).

${ }^{30}$ B. W. Ninham, T. T. Duignan, and D. F. Parsons, Curr. Opin. Colloid Interface Sci. 16, 612 (2011).

${ }^{31}$ A. Salis and B. W. Ninham, Chem. Soc. Rev. 43, 7358 (2014).

${ }^{32}$ M. Biesalski, D. Johannsmann, and J. Rühe, J. Chem. Phys. 120, 8807 (2004).

${ }^{33}$ G. Liu and G. Zhang, QCM-D Studies on Polymer Behavior at Interfaces, Springer Briefs in Molecular Science (Springer, 2013).

${ }^{34}$ O. Azzaroni, S. Moya, T. Farhan, A. A. Brown, and W. T. S. Huck, Macromolecules 38, 10192 (2005).

${ }^{35}$ R. Kou, J. Zhang, T. Wang, and G. Liu, Langmuir 31, 10461 (2015).

${ }^{36}$ A. Striegel, W. W. Yau, J. J. Kirkland, and D. D. Bly, Modern Size-Exclusion Liquid Chromatography: Practice of Gel Permeation and Gel Filtration Chromatography, 2nd ed. (John Wiley \& Sons, Inc., 2009).

${ }^{37}$ S. Tayyab, S. Qamar, and M. Islam, Biochem. Educ. 19, 149 (1991).

${ }^{38}$ V. S. J. Craig and M. Plunkett, J. Colloid Interface Sci. 262, 126 (2003).

${ }^{39}$ S. Edmondson, V. L. Osborne, and W. T. S. Huck, Chem. Soc. Rev. 33, 14 (2004).

${ }^{40}$ W. S. Harris, "Electrochemical studies in cyclic esters," Ph.D. thesis, University of California, Radiation Laboratory, Berkeley, CA, 1958. 
${ }^{41}$ C.-M. Yu, S. Mun, and N.-H. L. Wang, J. Chromatogr. A 1132, 99 (2006)

${ }^{42}$ R. Kou, J. Zhang, Z. Chen, and G. Liu, "Counterion specificity of polyelectrolyte brushes: Role of the specific ion-pairing interactions," ChemPhysChem (submitted).

${ }^{43}$ V. S. Craig, B. W. Ninham, and R. M. Pashley, J. Phys. Chem. 97, 10192 (1993).

${ }^{44}$ V. S. J. Craig, B. W. Ninham, and R. M. Pashley, Nature 364, 317 (1993).

${ }^{45}$ J. Lyklema, Adv. Colloid Interface Sci. 100, 1 (2003).
${ }^{46}$ W. Kunz and R. Neueder, in Specific Ion Effects, edited by W. Kunz (World Scientific Publishing Co. Pte. Ltd., 2009), Chap. 1, pp. 10-11.

${ }^{47} \mathrm{P}$. Zuman and S. Wawzonek, in The Chemistry of Nonaqueous Solvents, edited by J. J. Lagowski (Academic Press, 1978), Vol. VA, pp. 121-143.

${ }^{48}$ J. N. Butler, D. R. Cogley, and E. Grunwald, J. Phys. Chem. 75, 1477 (1971).

${ }^{49}$ Y. Marcus, J. Solution Chem. 33, 549 (2004).

${ }^{50}$ Y. Marcus, J. Solution Chem. 34, 317 (2005). 